\title{
Optimal distribution feeder reconfiguration with optimal planning of distributed generation for loss reduction and voltage improvement using differential evolution algorithm
}

\author{
Ahmad B. Ghaweta* and Yuan Liao \\ Department of Electrical and Computer Engineering, \\ University of Kentucky, \\ Lexington, Kentucky 40506, USA \\ Email: Agh228@g.uky.edu \\ Email: yuan.liao@uky.edu \\ *Corresponding author
}

\begin{abstract}
In the late years, there has been a noticeable influx of DGs onto the grid. This shift alters the manner in which electricity is being generated, transmitted, and managed. DGs conflicts have already risen between distribution systems designed for one-way power flow and DGs that want to force power flow in the opposite direction. Screening methods exist to avoid adverse impacts due to DGs, but this addressing the abundance of DGs interconnection requests and can result in higher overall costs if the resource is not fully integrated and located appropriately. A method to determine the optimal feeder reconfiguration and optimal allocation and size of DGs is proposed. The DG location is determined using the sensitivity of power losses. Then, the proposed differential evolution algorithm (DEA) is used to obtain the optimal size of the DG unit. The proposed algorithm is validated using the revised version of IEEE 33-bus radial distribution system.
\end{abstract}

Keywords: network reconfiguration; differential evolution algorithm; DEA; distributed generation; DG; sensitivity analysis; optimal size and location.

Reference to this paper should be made as follows: Ghaweta, A.B. and Liao, Y. (2019) 'Optimal distribution feeder reconfiguration with optimal planning of distributed generation for loss reduction and voltage improvement using differential evolution algorithm', Int. J. Forensic Software Engineering, Vol. 1, No. 1, pp.73-90.

Biographical notes: Ahmad B. Ghaweta received his BSc in Electrical and Computer Engineering from the University of Almergeb, Alkoms, Libya in 2003, his MSc in Electrical Engineering from Gannon University, Erie, Pennsylvania in 2011, and he is currently pursuing his $\mathrm{PhD}$ in Electrical Engineering from University of Kentucky, USA.

Yuan Liao is a Professor in the Department of Electrical and Computer Engineering at the University of Kentucky. He obtained his $\mathrm{PhD}$ in Electrical Engineering from Texas A\& M University, College Station, Texas in 2000. He worked as a Consulting R\&D Engineer and then Principal Consulting R\&D Engineer at the Corporate Research Center of ABB Inc., Raleigh, North Carolina from 2000 to 2005 . He has worked on projects in areas of fault location, fault type classification, high performance decomposition techniques for large scale resource scheduling optimisation, development of a competitive power market simulator-GridView, fault-tolerant NMS/SCADA, 
system expansion and distributed generation siting, asset management, power quality analysis, power system planning, and smart grid.

This paper is a revised and expanded version of a paper entitled 'Optimal distribution feeder reconfiguration with distributed generation using intelligent techniques' presented at Electro-information Technology Conference, Rochester MI, 2-3 May 2018.

\section{Introduction}

Due to the environmental impact concerns and incentives from regulators, distributed generation (DG) has become the central part of the distribution networks. DG is known as an on-site generation or decentralised generation. Its referred to the generation of electricity for use on-site, rather than transmitting energy over the electric grid from a large, centralised facility. That is to take its advantages of cleaner energy, less loss, and local power supply. The impact of DG by adding an active power source to the distribution network is aiming mainly to reduce the active power loss in the distribution network and as a result, improving the voltage profile at the network. Nowadays, these renewable DG are required to equip with reactive power devices (such as static VAR compensators, capacitor banks, etc.), to provide reactive power as well as to control the voltage at their terminal bus. DG's have various technical benefits such as voltage profile improvement, relief in feeder loading, power loss minimisation, stability improvement, and voltage deviation mitigation. In other words, the DG does not benefit the network if placed in any random location in the system. Therefore, it is crucial to find the optimal location and size of the DG. Most distribution networks are weak and radial with low short-circuits capacity. Therefore, there is a limit to which power can be injected into the distribution network without compromising the power quality and system stability. Thus, the differential evolution algorithm (DEA) is proposed to find the optimal location and size of the planned DGs units and demonstrating the system voltage within a defined boundary $[0.95-1.05$ p.u $]$.

The IEEE Standard 1547 has to be obeyed for the interconnection of distributed energy resource (DER). DER include distributed generators and energy storage systems. The standards provide requirements of connecting the DGs to the grid, relevant to the performance, operation, safety considerations, and maintenance of the interconnection. Such requirements are that, first, the DER units do not unintentionally provide power to the adjacent electricity customers or the utility grid when the grid has lost its power supply from the transmission system (Basso, 2014). Second, in the case of an open-phase condition, the DGs must detect and stop injecting power to the grid. Third, integrated DGs units must coordinate with utility voltage regulators and protection devices. DGs should have the ability to change its output active and reactive power in order to contribute in regulating the network voltages.

To study the impact of integrating DGs units into the distribution network, several kinds of literature have proposed different optimisation techniques. The optimum placement and sizing are performed to achieve different objectives. In De Souza and De Albuquerque (2006) evolutionary programming with the objective of maximising the reduction of the load supply costs was used. Besides, Siano (2007) proposed the 
combination of a genetic algorithm (GA) and optimal power flow (OPF) to efficiently site and size a predefined number of DGs. It differs from other proposed methods that only define the optimal locations and capacities of DG as a means of ensuring that the maximum amount of DG can be connected to existing and future networks. Also, in Witchit (2006) particles swarm optimisation (PSO) was used for optimal placement of multi-DGs with the aim of minimising the total real power loss, other various methods such as simulated annealing technique (Aly et al., 2010), Tabu search method (Maciel and Padilha-Feltrin, 2009; Gandomkar et al., 2005), artificial bee colony (ABC) (Abu-Mouti and El-Hawary, 2011), etc. Zhu (2006) introduced the use of time-varying loads for analysis of reliability and efficiency of distribution networks with DG. In Ochoa (2008) proposed a time-varying approach in demand load and generation, and steady-state analysis of technical issues such as losses, voltages, reserve capacity of conductors, and short-circuit levels was presented.

The proposed DEA is to investigate the impact of the DG installation on the performance of the distribution network and its parameters such as node voltages, active and reactive power loss. The topology is that the DG unit is injecting real power to the selected node and as a result, the voltage of this particular node will rise. Therefore, the network constraints (e.g., nodes voltage and line capacity) must be checked to prevent from having overvoltage event or exceeding line capacity limits. Only the feasible solution that can satisfy all the operation constraints is considered. The solution methodology herein is to evaluate the system without DG connection and then reconfigure the system by closing the tie switches and specify the tie with the maximum voltage difference and consider closing this tie first, then the DG location is obtained using the sensitivity of power losses with respect to real power injection at each bus. The most sensitive bus is selected for installing the DG unit. Because the integration of the DG adds positive real power injections, the optimal location is the one with the most negative sensitivity in order to obtain the largest power loss reduction. After the location is specified the proposed DEA is used to obtain the optimal size of the DG unit.

The proposed technique will minimise real power losses and improve the system voltage profile. The optimisations techniques are performed on three stages; first, network reconfiguration is performed, then, the optimal location of the planned DG is determined, and finally, the size of DG located at its optimal location is obtained by using the proposed DEA. Differential evolution (DE) is a stochastic, population-based search strategy developed by Price et al. (2005). While DE shares similarities with other evolutionary algorithms (EAs), it differs significantly in the sense that information from the current population is used to guide the search process. In the DE, all solutions have an equal opportunity of being preferred as parents, and selection does not depend on their fitness values. In DE, each new solution fashioned competes with its parent, and the superior one wins the contest (Kumar et al., 2014). In DE operation the child vector is generated by applying the mutation and crossover operation. The mutation procedure expands the search space. In mutation operation, a trial vector is generated with the help of the objective vector and two erratically preferred individuals. The deviation in the objective vector depends on mutation factor $\mathrm{F}$ and the difference between the randomly selected individuals.

On the other hand, the crossover operation is applied between the objective vector and parent vector to generate the child vector using the crossover probability (CR). Like any other method, these tuning factors have a boundary such that large values may result 
in skipping of actual solutions, and small values also may cause performance degrades. The conflicts in the child vector from the parent vector depends on the values of $F$ and CR.

The remainder of this paper is structured as follows: Section 2 represents the problem formulation of power loss minimisation. Section 3 presents the proposed DEA. Section 4 provides the approach to find the optimal placement and size of DG units. Section 5 describes the test system. Simulation results and discussion are given in Section 6 . Finally, conclusions are provided in Section 7.

\section{Problem formulation}

The problem considered herein is to minimise the system power losses and to improve the voltage profile. The integration of single or multiple DGs units in the power distribution network is a challenging problem that involves complex nonlinear equations. Sizing and allocation of the DGs are a vital parameter as the random placement of DG in the grid can cause a negative consequence such as an increase in system total power losses and out-of-limit voltages. On the contrary, if the DGs are precisely placed and optimally sized, then it will improve the system's overall efficiency. This work mainly focuses on identifying the optimal size and location of installing DGs units in the grid in the way all the constraints are satisfied. The problem is formulated as a multi-objective problem, whose objectives include minimisation of total system active and reactive power losses, minimisation of feeder's currents imbalance, and minimisation of node voltage deviation considering the substation as a reference.

The terms in the objective function are focused on reducing power losses and improving the voltage profile. Once the DG size is optimised, the network is reconfigured to achieve the desired objectives. The objective of the network reconfiguration is based on real power loss reduction. The loss minimisation is formulated as:

$$
\min P_{\text {loss }}(x)
$$

with

$$
P_{\text {loss }}=\sum_{i=1}^{N b} R_{i} *\left|I_{i}\right|^{2}
$$

such that,

$$
\begin{array}{ll}
I_{i} \leq I_{\max } & \forall i=1,2, \ldots, N b \\
V_{\min } \leq V_{j} \leq V_{\max } & \forall j=1,2, \ldots, N
\end{array}
$$

where

$P_{\text {loss }} \quad$ total line losses of the feeder

$x \quad$ status vector of the switch

$\mathrm{Nb}$ total number of branches in the whole system

$I_{i} \quad$ current magnitude of branch $i$ 
$I_{\max } \quad$ upper limit of branch current magnitude

$V_{j} \quad$ voltage magnitude of branch $j$

$V_{\min }, V_{\max } \quad$ lower and upper limit of node voltage magnitude, herein; $0.95 \leq V_{j} \leq 1.05$

$R_{i} \quad$ resistance of branch $i$

$N \quad$ total number of buses.

$$
x=\left[s w_{1}, s w_{2}, s w_{3}, \ldots, s w_{\text {number of variables }}\right]
$$

The total power loss of each branch is determined by summing up all the losses of all branch sections of the feeder as follows:

$$
\begin{aligned}
& \text { Delta }_{p}=\sum_{\text {branch }=1}^{N b} I_{\text {branch }}^{2} * R_{\text {branch }} \\
& \text { Delta }_{Q}=\sum_{\text {branch }=1}^{N b} I_{\text {branch }}^{2} * X_{\text {branch }}
\end{aligned}
$$

where

Delta $_{p} \quad$ real power loss.

Delta $_{Q}$ reactive power loss.

The operating constraints of the system are the inequality and equality constraints.

\subsection{Inequality constraints}

- $\quad$ Power generation limits

The output generating power of the DGs unit must be within a limit.

Thus,

$$
\begin{aligned}
& P_{D G i, \text { min }} \leq P_{D G i} \leq P_{D G i, \text { max }} \\
& Q_{D G i, \text { min }} \leq Q_{D G i} \leq Q_{D G i, \text { max }}
\end{aligned}
$$

- Branch power limit constraint:

$$
\begin{gathered}
P_{j}^{2}+Q_{j}^{2} \leq\left(S_{j}^{\max }\right) \\
P_{D G i}^{\min } \leq P_{D G i} \leq P_{D G i}^{\max } \\
Q_{D G i}^{\min } \leq Q_{D G i} \leq Q_{D G i}^{\max }
\end{gathered}
$$

- Bus voltage limits

$$
V_{\min } \leq V_{i} \leq V_{\max }
$$

- Branch current magnitude: maximum limit

$$
\left|I_{i}\right| \leq I_{\max }
$$




\subsection{Equality constraints}

Power balance: the power flow equations must be satisfied

$$
\begin{aligned}
P_{i} & =P_{D G i}-P_{D i} \\
Q_{i} & =Q_{D G i}-Q_{D i}
\end{aligned}
$$

where $P_{D G i}$ and $Q_{D G i}$ are distributed power generation at bus $i . P_{D i}$ and $Q_{D i}$ are the loads at bus $i$.

In this paper, the feasible solution is obtained in such a way that optimal location and size of the connected DG satisfies the operating constraints, by taking into account that all the node voltages are within limits and that there are no violations in the value of voltage.

\section{Methodology}

This paper proposes a DEA approach based on our previous work presented in Ghaweta and Liao (2018) to solve the feeder reconfiguration problem and the impact of DG power simultaneously. Figure 1 shows the flowchart of the proposed approach to reconfigure and find the optimal location and size of the planned DGs units.

DEA involves three unique advantages: independence on the initial population values in finding the global minima, using few parameters, and fast convergence.

DE differs from other algorithms such that:

1 Mutation is applied first to generate a trial vector, which is then used within the crossover operator to produce one offspring.

2 Mutation step sizes are not sampled from a prior known probability distribution function.

3 In DE, mutation step sizes are influenced by differences between individuals of the current population.

For an objective function $f: X \subseteq \mathbb{R}^{D} \rightarrow \mathbb{R}$ where the feasible region $X \neq \varnothing$ the minimisation problem is to find $x^{*} \in X$ such that

$$
f\left(x^{*}\right) \leq f(x) \quad \forall x \in X
$$

such that $f\left(x^{*}\right) \neq-\infty$.

The parameter vectors of the optimisation function have the form:

$$
x_{i, G}=\left[x_{1, i, G}, x_{2, i, G}, \ldots, \ldots, x_{D, i, G}\right] \quad \forall i=1,2, \ldots, N
$$

where

$N$ population size

$G$ generation number

$D$ D-dimensional search space. 
Figure 1 Flowchart of DE's generate-and-test loop

1) Choose the target vector and best vector

2) Random choice of two population members

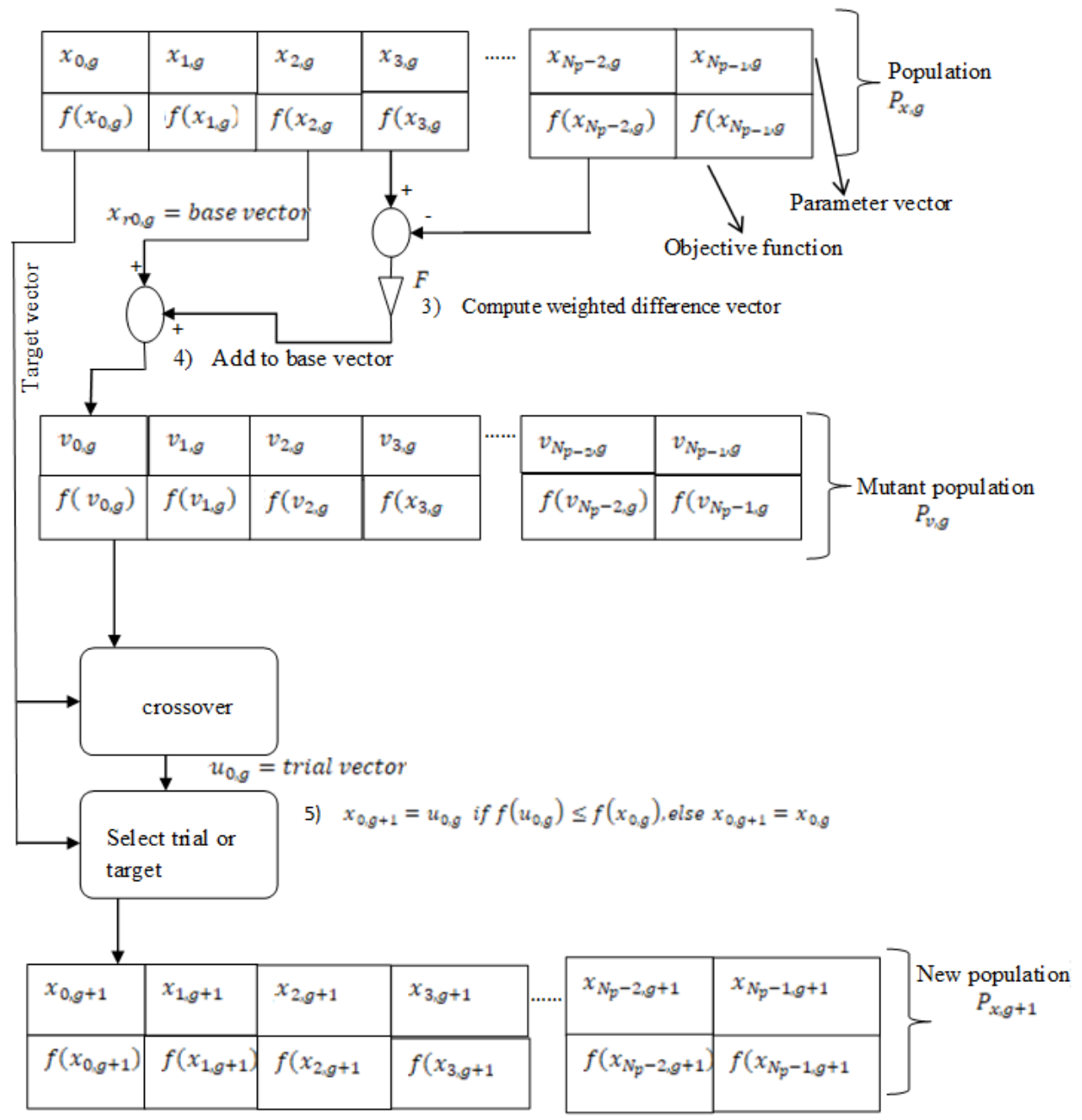

\subsection{Initialisation}

The initialisation procedure is to define the upper and lower bounds of each parameter such that

$$
x_{j}^{L} \leq x_{j, i, 1} \leq x_{j}^{U}
$$

Then randomly select the initial parameters values uniformly on the intervals $\left[x_{j}^{L}, x_{j}^{U}\right]$. 
The population matrix is formulated based on the number of variables (e.g., number of open ties) and maximum population size.

$$
\text { pop }_{\text {matrix }}=\left[\begin{array}{ccccc}
x_{1} & x_{2} & \ldots & \ldots & x_{n} \\
& & \vdots & & \\
& & \vdots & & \\
& & \vdots & & \\
x_{1 p} & x_{2 p} & \ldots & \ldots & x_{n p}
\end{array}\right]
$$

where the $n$ denoted number of variables, and $p$ is the population size.

\subsection{Statistics}

Load-flow computation (1) is used as a fitness function by adding some constraints such as

$$
F=P_{\text {loss }}\left(S_{v}\right)+\sum_{i=1}^{N b} \lambda_{I, i} *\left(I_{i}-I_{\lim }\right)^{2}+\sum_{j=1}^{N} \lambda_{v, j} *\left(V_{j}-V_{\lim }\right)^{2}
$$

where $\lambda_{I, i}$ and $\lambda_{V, j}$ are the penalty factors that can be adjusted in the optimisation procedure. $I_{\text {lim }}$ and $V_{\text {lim }}$ are defined as

$$
\begin{gathered}
I_{\lim }= \begin{cases}I_{i} & \text { if } I_{i} \leq I_{\max } \\
I_{\max } & \text { if } I_{i}>I_{\max }\end{cases} \\
V_{\lim }= \begin{cases}V_{j} & \text { if } V_{\min } \leq V_{j} \leq V_{\max } \\
V_{\min } & \text { if } V_{j}<V_{\max } \\
V_{\max } & \text { if } V_{j}>V_{\max }\end{cases}
\end{gathered}
$$

\subsection{Mutation}

One of the DE advantages is searching from a large search space, and the mutation procedure expands the search space. Each of the $N$ parameter vectors undergoes mutation, recombination, and selection. To illustrate that, for a given parameter vector $x_{i, G}$ randomly selects three vectors $x_{r 1, G}, x_{r 2, G}$ and $x_{r 3, G}$ such that the indices $i, r 1, r 2$ and $r 3$ are distinct. Then calculate the donor vector by adding the weighted difference of two of the vectors to the third such that:

$$
v_{i, G+1}=x_{r 1, G}+F\left(x_{r 2, G}-x_{r 3, G}\right)
$$

where $F$ is the mutation scale factor.

The scale factor, $F \in(0, \infty)$, is a positive real number that controls the amplification of the differential variations, $\left(x_{r 2, G}-x_{r 3, G}\right)$. The smaller the value of $F$ the smaller the mutation step sizes, and the longer it will be for the algorithm to converge. Larger values 
for $F$ facilitate exploration, but may cause the algorithm to overshoot good optima. The value of $F$ should be small enough to allow differentials to explore tight valleys, and large enough to maintain diversity. As the population size increases, the scaling factor should decrease.

\subsection{Recombination}

Recombination incorporates successful solutions from the previous generation. The trial vector $u_{i, G+1}$ is developed from the elements of the target vector, $x_{i, G}$ and the elements of the donor vector, $v_{i, G+1}$. Then, the elements of the donor vector enter the trial vector with the probability $C R$.

Thus,

$$
u_{j, i, G+1}= \begin{cases}v_{j, i, G+1} & \text { if } \operatorname{rand}_{j, i} \leq C R \text { or } j=I_{\text {rand }} \\ x_{j, i, G} & \text { if } \text { rand }_{j, i}>C R \text { or } j \neq I_{\text {rand }}\end{cases}
$$

where $i=1,2, \ldots, N ; j=1,2, \ldots, D$.

Where $\operatorname{rand}_{j}, i \sim U[0,1], I_{\text {rand }}$ is a random integer from $[1,2 \ldots, D]$, and $I_{\text {rand }}$ ensures that $v_{i, G+1} \neq x_{i, G}$. The probability of recombination $C R$, has a direct influence on the diversity of DE. This parameter controls the number of elements of the parent, $x i(t)$, that will change. The higher the probability of recombination, the more variation is introduced in the new population, thereby increasing diversity and increasing exploration. Increasing $C R$ often results in faster convergence, while decreasing $C R$ increases search robustness (Joshi and Sanderson, 1997; Lopez Cruz et al., 2013).

\section{Optimum placement and sizing of DGs}

Power loss reduction problems are depending on the network topology and the number of connected DGs, location, the output power of the DGs units installed in the grid. All the connected DGs must obey the IEEE Standard 1547. Also, it can be aggregated into an equivalent unit.

\subsection{Optimal location of DG units}

The DG location is obtained using the sensitivity of power losses with respect to real power injection at each bus; the most sensitive bus is selected for installing the DG unit. Because the integration of the DG adds positive real power injections, the bus with the highest negative sensitivity value is considered as the weakest bus in the system, and DG unit will be placed at that respective bus. The mathematical formulation considered in the optimal placement is the maximum of equation (3), more details can be found at Ding and Loparo (2016) and Das et al. (2016), and is given as:

$$
D G_{\text {location }}=\max \left(\operatorname{Delta}_{p}(\mathrm{Nb})\right)
$$




\subsection{The optimum size of $D G$ units}

The optimum sizes of the DG units to be installed at the obtained optimal location are determined to minimise system total power losses and improve the voltage profile. Thus, for a reconfigurable system with connected DG units, the solution strategy is to generate a set of random DG sizes within a specified range using the proposed DEA. Placed at the obtained location (14) and then for each size of DG the power flow is executed. Evaluate the fitness function for total power reduction, and the feasible solution is obtained.

The steps of network reconfiguration, DG location and sizing with the DEA are explained as follows:

Step 1 Read system data.

Step 2 Run power flow without DG connection.

Step 3 Determine the DG location using equation (14).

Step 4 By using DEA do the following:

1 reconfigure the system following our method in Ghaweta and Liao (2018).

2 generate a random size of DG using the specified minimum and maximum boundaries of the DG size.

Then, evaluate the fitness function of each size and check all the constraints and all the node voltages, memorise the feasible solutions and write down the optimal solution and do the same for another switch status.

\section{Test system description}

The effectiveness of the proposed DEA to solve the optimal size and location of the DGs units followed by the network reconfiguration is illustrated using the IEEE 33-bus radial distribution test feeder (Kersting, 1991). The test system is shown in Figure 2. This test system is presented on a per phase basis, and the loads along the feeder are considered as a spot load with constant $P, Q$ loads placed at the end of the lines. In addition, each line in the system is associated with a sectionalised switch. This system has five tie-switches (dotted lines) numbered as $(33,34,35,36,37)$ and 32 in service branches (solid lines) and 33 nodes. The system has a total load of 3.715 MW and 2.30 MVAR without any connected DG unit. Base values are $12.66 \mathrm{kV}$ and 100 MVA respectively.

Figure 3 illustrated the mesh structure of the IEEE 33-bus test system. The total number of meshes is equal to the number of normally open tie switches. Formulating the meshed network is crucial because it will benefit the construction of the population matrix. Additionally, there are some conditions of constructing the meshed network such as:

1 There should be no common switches between two meshes.

2 Only one open switch in each mesh.

3 Switches of the end node loads remain on and should not be considered in the boundary.

4 The radial structure must be retained. 
Figure 2 Single line diagram of IEEE 33-bus distribution system, $s$ denoted switch and the dotted lines represent the tie switches (see online version for colours)

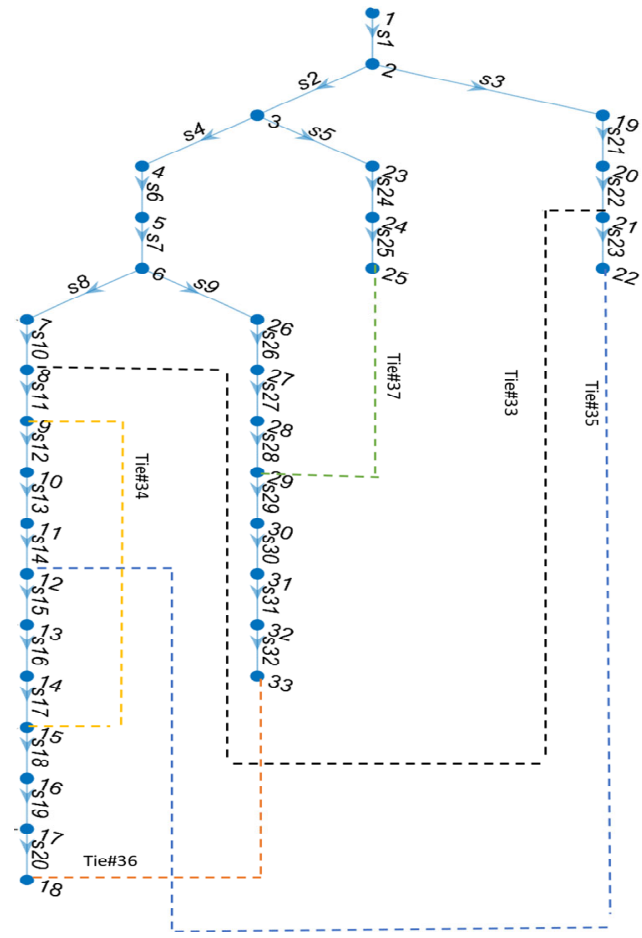

Figure 3 Mesh formulation of the 33-bus test system (see online version for colours)

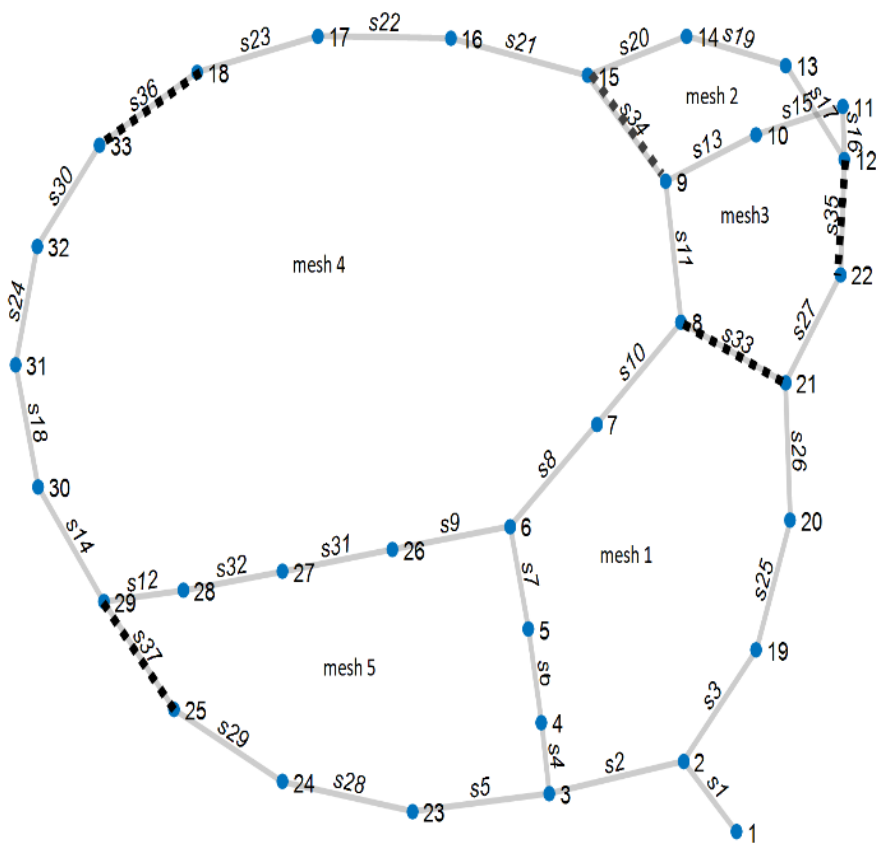




\section{Simulation results and discussion}

The most desired objective of the proposed approach is to determine the optimal location and size of the planned DGs units. The real power and reactive power losses, as well as the voltage profile enhancement, are the primary goals of this work. Table 1 shows the simulation results of deferent cases for single and multiple DGs units installed at their optimal location and size. Figures 3, 4, 5, 6, 7, 8 and 9 present the simulation results of the case studies. As it can be noticed, there has been a significant reduction of the real and reactive power losses after installing the DGs units and network reconfiguration. The system node voltages are also improved and well constrained between the limits, also feeder current which is the current from the substation also been reduced after installing the DGs units. There has also been a significant achievement in mitigating the node Voltage deviation. The maximum and minimum boundary of the DGs size is set between $50 \mathrm{~kW}$ to $1,000 \mathrm{~kW}$. Basically, for each case where the installed DGs units inject power to the system, the node voltage limit is checked. In fact, the voltage magnitude of the connected DG node will rise and as a result it will affect the downstream voltages and potentially will affect the upstream as well. Then the nearby voltage regulator must act to handle the voltage variation. Thus, the connected DG unit must contribute and have the ability to change its output power in order to absorb or inject additional reactive power to accommodate the voltage variation. If a voltage violation occurs, then the solution is considered to be infeasible. The algorithm will consider another size and location of DG and search for only the feasible solutions where all the constraints are satisfied.

Table 1 Simulation results of the optimal switching plane and optimal placement and sizing of installed DG units

\begin{tabular}{|c|c|c|c|c|c|c|}
\hline Case \# & $\begin{array}{c}D G \\
\text { location }\end{array}$ & $D G$ size $(k W)$ & $\begin{array}{l}\text { Real } \\
\text { power } \\
\text { loss }\end{array}$ & $\begin{array}{c}\text { Reactive } \\
\text { power } \\
\text { loss }\end{array}$ & $\begin{array}{l}\text { Sub-current } \\
\text { (KA) }\end{array}$ & $\begin{array}{l}\text { Tie-switch } \\
\text { status }\end{array}$ \\
\hline $\begin{array}{l}1 \text { (base } \\
\text { case) }\end{array}$ & - & - & 64.3894 & 54.0280 & 345.1304 & $\begin{array}{c}33,34,35 \\
36,37\end{array}$ \\
\hline 2 & 33 & 324.8984 & 34.4397 & 26.2894 & 293.1455 & $\begin{array}{c}10,17,27, \\
18,32\end{array}$ \\
\hline 3 & 5,27 & $787.5761,882.4191$ & 44.9553 & 32.7910 & 196.2822 & $\begin{array}{c}10,19,27, \\
22,28\end{array}$ \\
\hline 4 & 19,33 & $683.7605,391.5140$ & 38.7450 & 31.7989 & 198.0507 & $\begin{array}{c}6,13,35 \\
18,9\end{array}$ \\
\hline 5 & $19,33,2$ & $\begin{array}{c}165.9004 \\
272.3896,380.0187\end{array}$ & 33.9801 & 26.2634 & 210.8594 & $\begin{array}{c}10,19,27, \\
18,32\end{array}$ \\
\hline 6 & $\begin{array}{c}2,23,5 \\
24\end{array}$ & $\begin{array}{c}735.9465 \\
487.3021 \\
825.1883,928.7449\end{array}$ & 35.9253 & 27.0175 & 279.2741 & $\begin{array}{c}26,20,35, \\
30,12\end{array}$ \\
\hline 7 & $\begin{array}{c}33,19, \\
23,20,2\end{array}$ & $\begin{array}{c}301.1961, \\
885.0333, \\
328.5141, \\
839.4796,642.0249\end{array}$ & 36.7124 & 31.2641 & 218.7507 & $\begin{array}{c}4,19,27 \\
24,32\end{array}$ \\
\hline 8 & $\begin{array}{l}23,2 \\
24,19 \\
22\end{array}$ & $\begin{array}{c}424.1403, \\
972.4571, \\
256.3604, \\
382.2527,273.1875\end{array}$ & 33.0319 & 25.5680 & 313.5803 & $\begin{array}{l}8,15,11 \\
23,31\end{array}$ \\
\hline
\end{tabular}


Different case studies have been considered herein, the base case is to run the system without DG connection and then reconfigure the system to compute all the branch currents and node voltages. Then, the branch with the maximum active power loss is considered first as an optimal location of installing the DG unit. The proposed method would randomly generate the DG sizes from the specified range $(50 \mathrm{~kW}$ to $1,000 \mathrm{~kW})$. The only size of the DG who satisfies all the operation constraints would be counted.

Table 2 Simulation parameters of the proposed DEA

\begin{tabular}{lcccccc}
\hline $\begin{array}{l}\text { Population } \\
\text { size }\end{array}$ & $\begin{array}{c}\text { Mutation } \\
\text { factor }\end{array}$ & $\begin{array}{c}\text { Crossover } \\
\text { rate }\end{array}$ & $\begin{array}{c}\text { Max } \\
\text { iteration }\end{array}$ & $\begin{array}{c}\text { Number of } \\
\text { variables }\end{array}$ & $\begin{array}{c}\text { Min DGs unit } \\
\text { size }(\mathrm{kW})\end{array}$ & $\begin{array}{c}\text { Max DGs unit } \\
\text { size }(\mathrm{kW})\end{array}$ \\
\hline 100 & 0.8 & 0.7 & 100 & 5 & 50 & 1,000 \\
\hline
\end{tabular}

Figure 4 Power flow results with DG unit installed at bus \# 33 (see online version for colours)
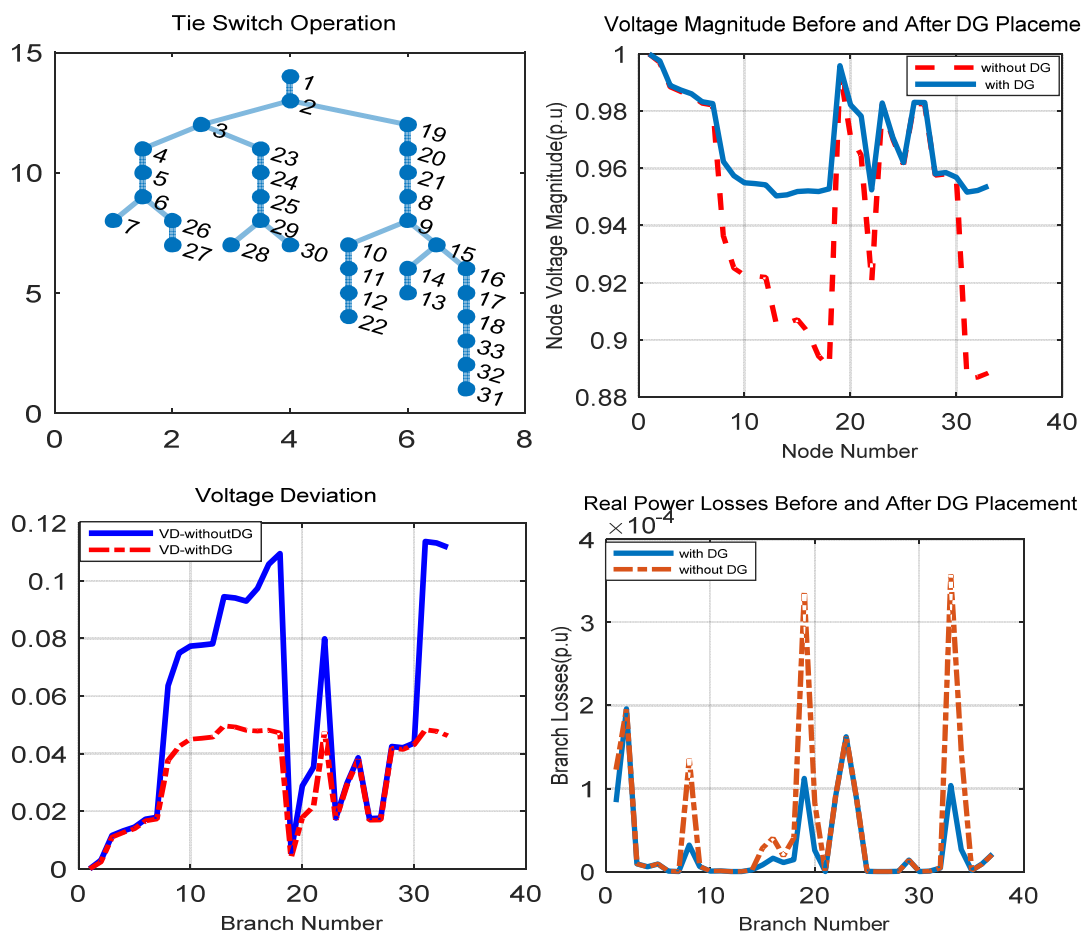

Simulation parameters of the proposed DEA are shown in Table 2. Mutation factor and crossover rate are the main tuning parameters. The new trial matrix is created from mutation and recombination procedure. Each trial is evaluated, and the obtained results are compared with the ones computed using the corresponding chromosome of the original population matrix. The best fitness is then saved and to be memorised. Figure 10 presents the performance of the proposed DEA. From the simulation results, the optimal minima were found in faster time and less iteration. The stopping criteria are that after each evaluation step the size of the population matrix is updated to prevent evaluating the same chromosome more than ones. 
Figure 5 Power flow results with DG units installed at bus \# 5 and 27 (see online version for colours)
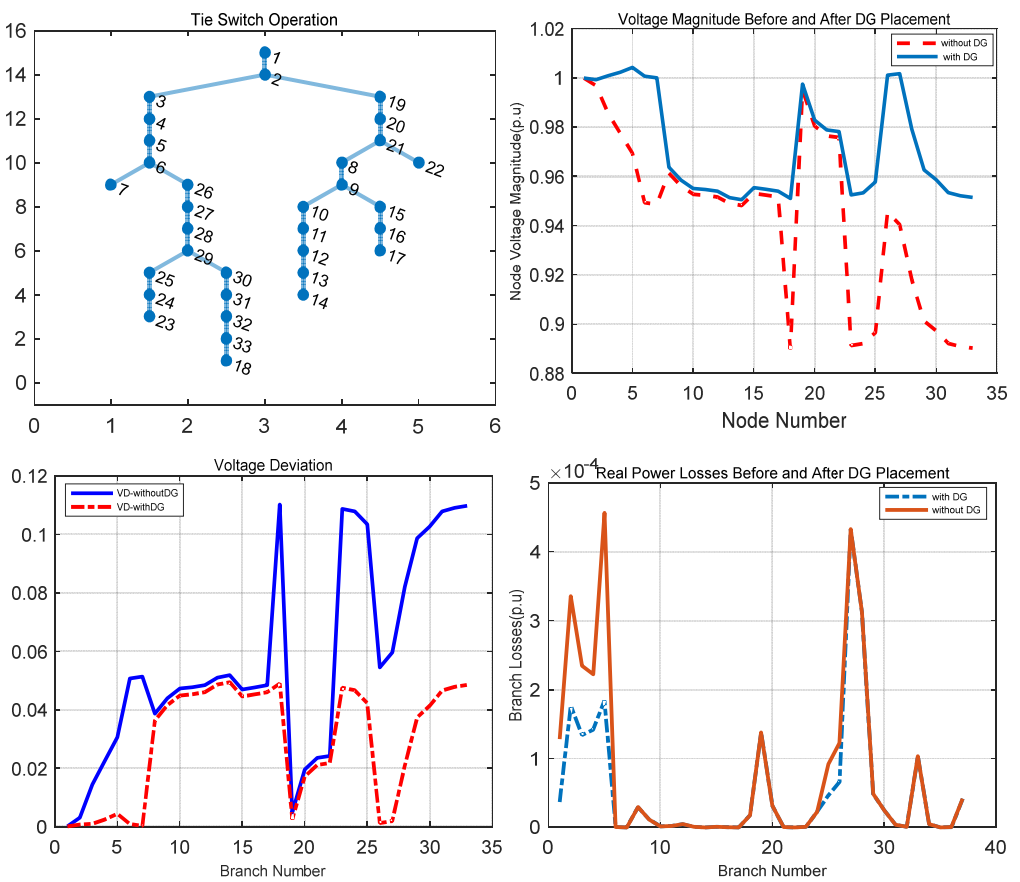

Figure 6 Power flow results with DG units installed at bus \# 19 and 33 (see online version for colours)
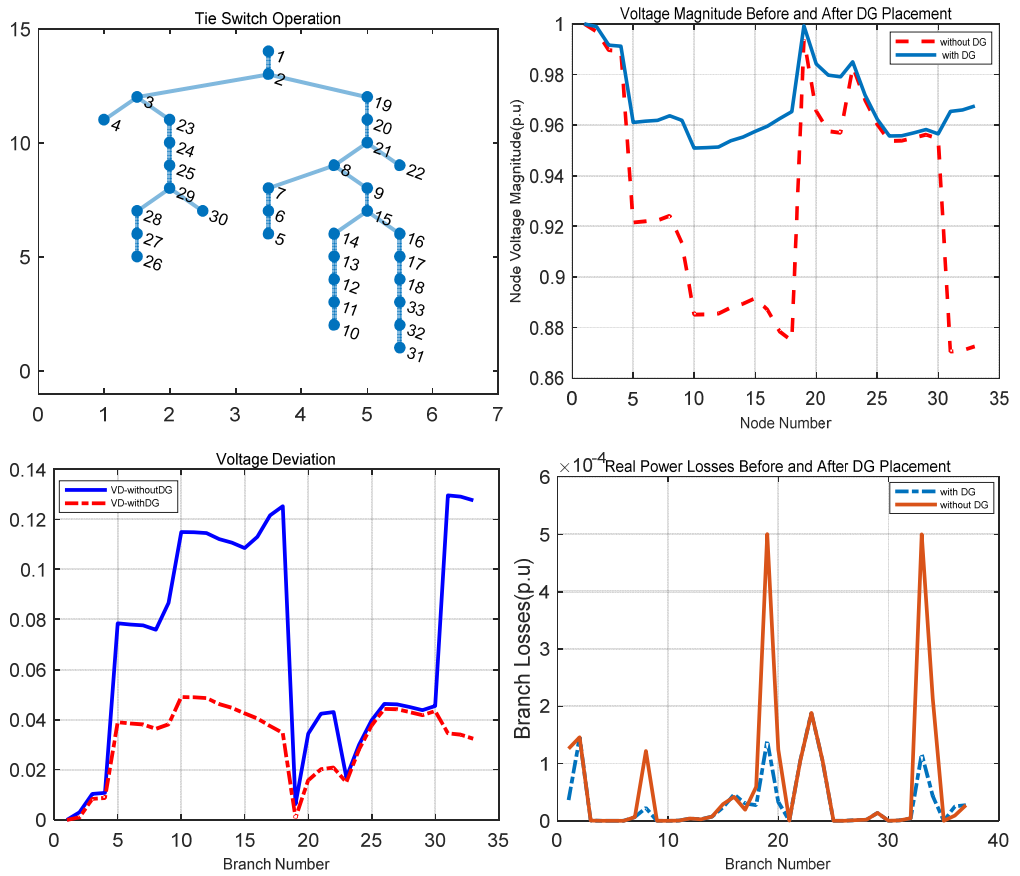
Figure 7 Power flow results with DG units installed at bus \# 19, 33 and 2 (see online version for colours)
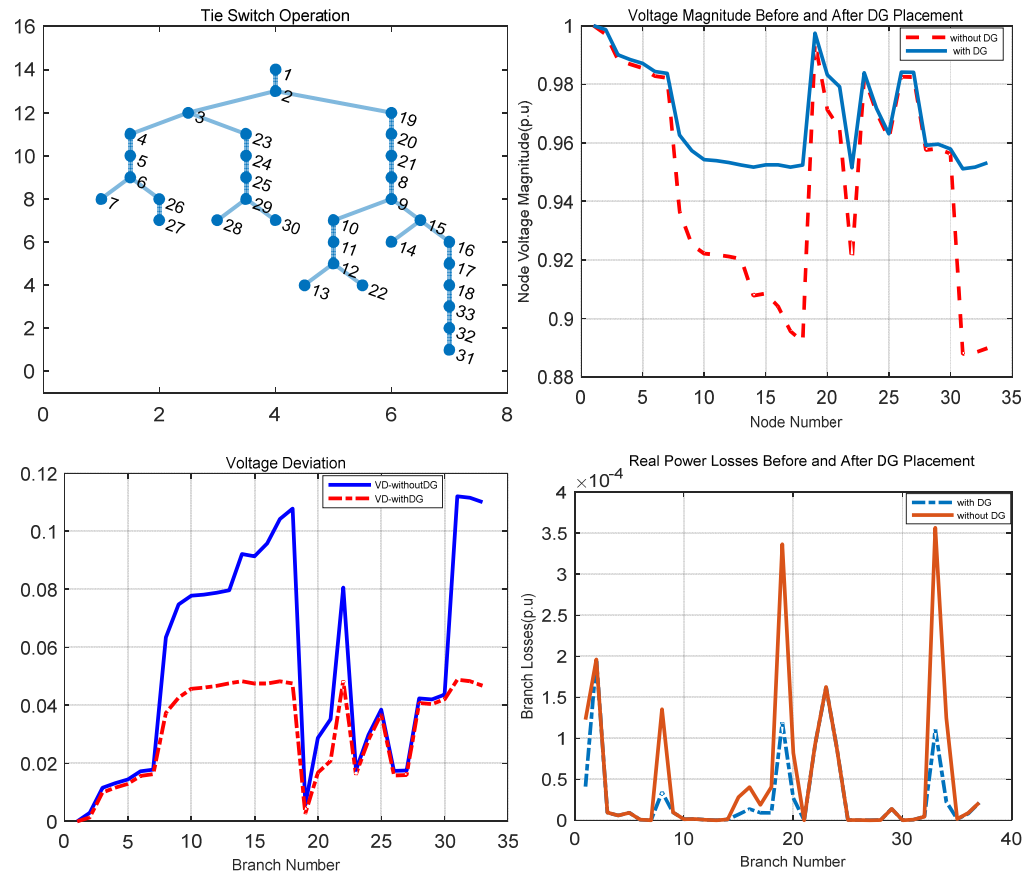

Figure 8 Power flow results with DG units installed at bus \# 2, 23, 5 and 24 (see online version for colours)
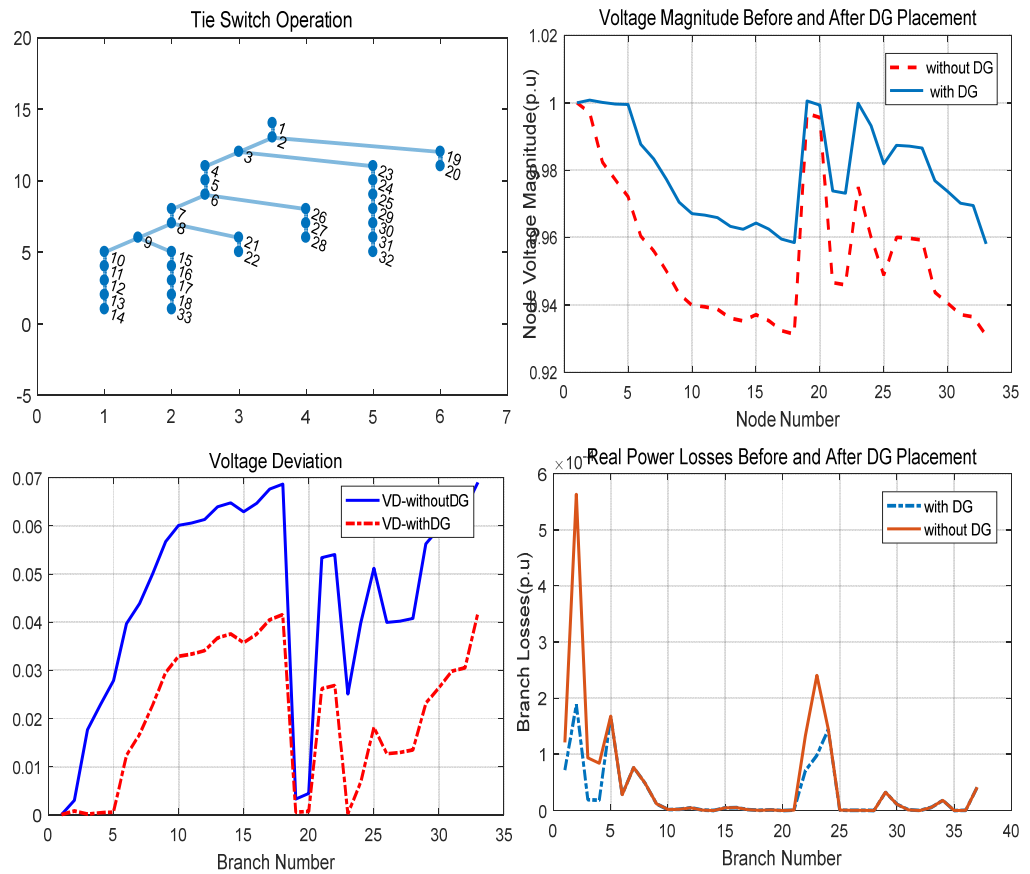
Figure 9 Power flow results with DG units installed at bus \# 33, 19, 23, 20 and 2 (see online version for colours)
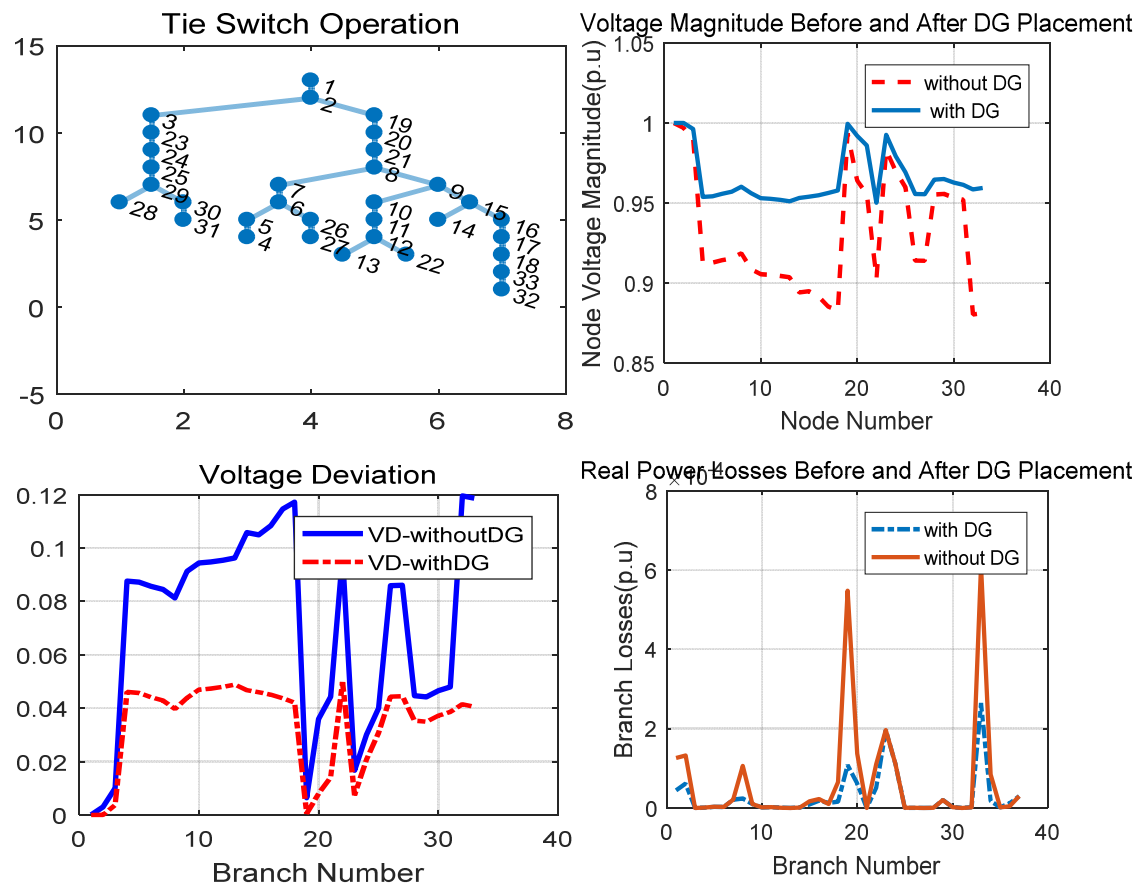

Figure 10 Power flow results with DG units installed at bus \# 23, 2, 24, 19, and 22 (see online version for colours)
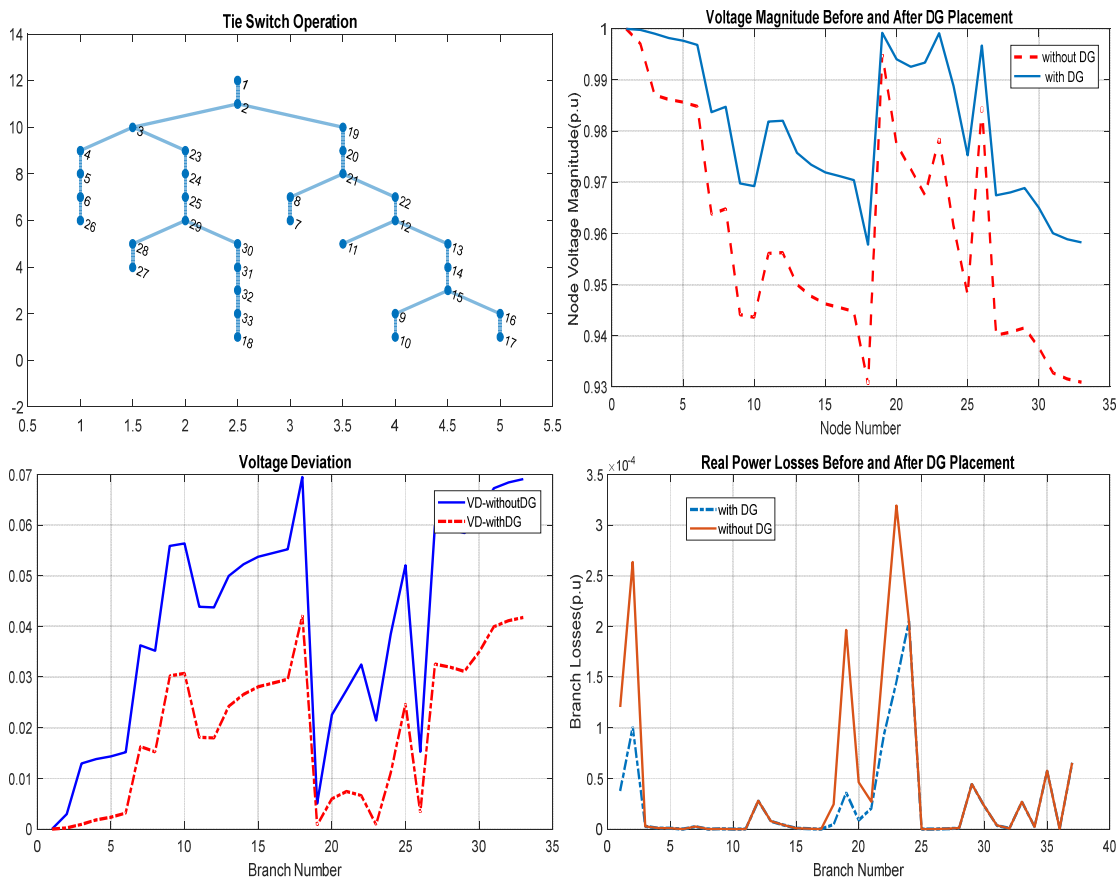
Figure 11 Convergence curve of the best fitness value of DEA (see online version for colours)

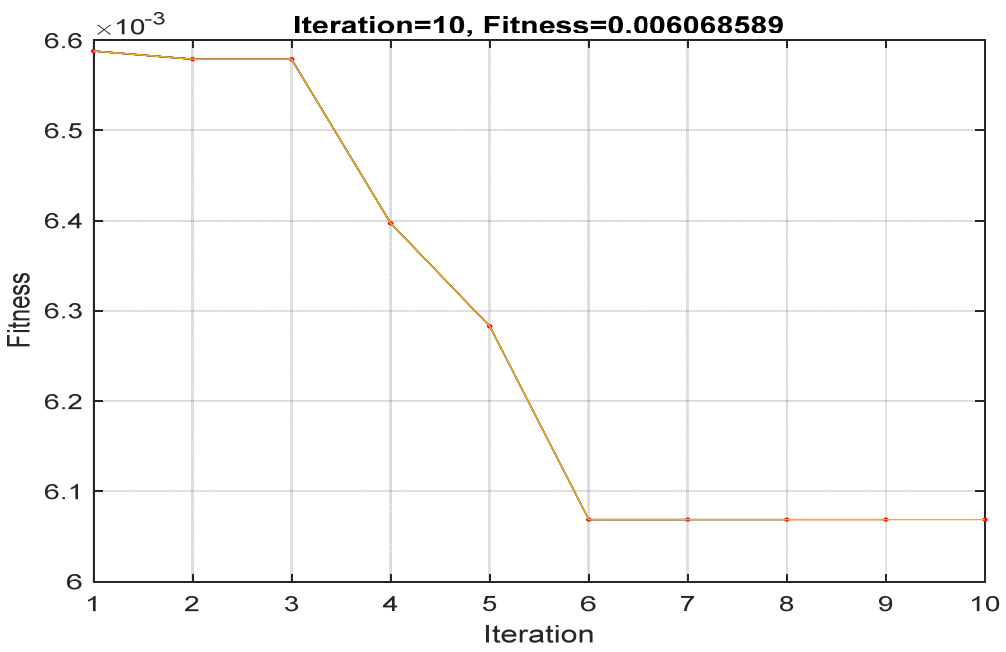

\section{Conclusions}

This paper proposed a new approach for distribution system reconfiguration and optimal sizing and placement of DGs units. The multi-objective problem is solved by using a DEA. This work is also a continuation of a recently published paper on optimal distribution feeder reconfiguration with DG using the intelligent technique. Size of the DGs units is determined using DEA, and the location is also obtained using sensitivity analysis based on the network real power loss. Different case scenarios have been studied including the integration of different numbers of DGs units. Simulation results of the IEEE 33-bus test system have demonstrated the efficiency and the effectiveness of the proposed algorithm for determining the optimal sizing and placement of the DGs units and reconfiguring the distribution feeders. The future work would be is to investigate the distribution feeder response characteristics. The distribution feeder response characteristics attentional is to determine the hosting capacity of the feeder. The hosting capacity of a feeder is defined as the amount of DERs a feeder can support under its existing topology, configuration, and physical response characteristics. If the hosting capacity is appropriately done then it will provide a range of information such as, how many DERs can be accommodated without system upgrades, what issues arise at the hosting capacity limits, the location of the DERs so that problems can be avoided, and the location where additional DERs are likely to cause issues on the grid. 


\section{References}

Abu-Mouti, F.S. and El-Hawary, M.E. (2011) 'Optimal distributed generation allocation and sizing in distribution systems via artificial bee colony algorithm', in IEEE Trans. on Power Del., Vol. 26, No. 4, pp.2090-2101.

Aly, A.I., Hegazy, Y.G. and Alsharkawy, M.A. (2010) 'A simulated annealing algorithm for multi-objective distributed generation planning', in Proceedings of IEEE PES Transmission and Distribution.

Baran, M.E. and Wu, F.F. (1989) 'Network reconfiguration in distribution systems for loss reduction and load balancing', in IEEE Transactions on Power Delivery.

Basso, T. (2014) IEEE 1547 and 2030 Standards for Distributed Energy Resources Interconnection and Interoperability with the Electricity Grid, National Renewable Energy Laboratory, December.

Das, S., Das, D. and Patra, A. (2016) 'Distribution network reconfiguration using distributed generation unit considering variations of load', in IEEE International Conf. Power Deliv. (ICPEICES).

De Souza, B.A. and De Albuquerque, J.M.C. (2006) 'Optimal placement of distributed generators networks using evolutionary programming', in Proceedings of IEEE PES Transmission and Distribution, Latin American, Venezuela.

Ding, F. and Loparo, K.A. (2016) 'Feeder reconfiguration for unbalanced distribution systems with distributed generation: a hierarchical decentralized approach', in IEEE Power and Energy Society General Meeting (PESGM), Cleveland.

Gandomkar, M., Vakilian, M. and Ehsan, M. (2005) 'A genetic-based Tabu search algorithm for optimal DG allocation in distribution networks', in Electric Power Components and System, Vol. 33, No. 12, pp.1351-1363.

Ghaweta, A. and Liao, Y. (2018) 'Optimal distribution feeder reconfiguration with distributed generation using intelligent techniques', in Electro-Information Technology Conference, Rochester, MI.

Joshi, R. and Sanderson, A.C. (1997) 'Minimal representation multisensor fusion using differential evolution', in the International Symposium on Computational Intelligence in Robotics and Automation, pp.255-273.

Kersting, W. (1991) 'Radial distribution test feeders', in IEEE Trans. Power Syst.

Kumar, S., Sharma, V.K. and Kumari, R. (2014) 'Memetic search in differential evolution algorithm', in International Journal of Computer Applications, March, Vol. 90, No. 6, pp.975-8887.

Lopez Cruz, I.L., Van Willigenburg, L.G. and Van Straten, G. (2003) 'Efficient differential evolution algorithms for multimodal optimal control problems', in Applied Soft Computing, Vol. 3, No. 2, pp.97-122.

Maciel, R.S. and Padilha-Feltrin, A. (2009) 'Distributed generation impact evaluation using a multi-objective Tabu search', in 15th International, November.

Ochoa, P.H. (2008) 'Evaluating distributed time-varying generation through a multiobjective index', in IEEE Transactions on Power Delivery, Vol. 23, No. 2, pp.1132-1138.

Price, K., Storn, R.M. and Lampinen, J.A. (2005) 'A practical approach to global optimization', Natural Computing Series, München, Germany.

Siano, H. (2007) 'Strategic placement of distributed generation capacity', in Proceeding of 19th International Conference on Electricity, Vienna.

Witchit, O. (2006) 'Optimal placement of distributed generation using particle swarm optimization', in Proceedings of the Australasian Universities Power Engineering, Melbourne, Victoria, Australia.

Zhu, B. (2006) 'Impact of DG placement on reliability and efficiency with time-varying loads', in IEEE Trans. Power Syst., Vol. 21, No. 1, pp.419-427. 